BULL. AUSTRAL. MATH. SOC.

VOL. $31(1985), 35-40$.

\title{
A REMARK ON COMPLETELY REDUCIBLE INEAR-RINGS
}

\section{Stefania De Stefano and Simonetta Di Sieno}

\begin{abstract}
A characterization of the completely reducible (zerosymmetric right) near-rings which are the direct sum of their socle of type 2 and of their 2-radical is given.
\end{abstract}

\section{Completely reducible near-rings}

Throughout this note $N$ indicates a zerosymmetric right near-ring; terminology and notation are those of [5]. In particular a non-zero left ideal $L$ of $N$ will be called minimal if it does not contain proper left ideals of $N$; simple if the $N$-group $L$ has no proper ideals; of type 2 if the $N$-group $L$ is monogenic and has no proper $N$-subgroups.

It is well known that if the near-ring $N$ is completely reducible that is, it is the sum of its minimal left ideals - then every minimal left ideal of $N$ is a simple one, so that $N$ is actually the direct sum of (a few of) its simple left ideals.

Assume that $N$ has at least one left ideal of type 2 ; then the socle of type 2 - that is the sum $\zeta_{2}(N)$ of all left ideals of type 2 of $N$ - is a non-zero left ideal of $N$ and therefore is a direct summand of $N$ (see [1], Lemma 1.3). Moreover the following result holds.

PROPOSITION 1. A completely reducible near-ming $N$ is the direct sum of its socle of type 2 and of a left ideal which is contained in the

Received 23 July 1984.

Copyright Clearance Centre, Inc. Serial-fee code: 0004-9727/85 $\$ A 2.00+0.00$. 
annihizator of the $N$-group $\zeta_{2}(N)$.

Proof. The near-ring $I N$ can be written as the direct sum of $\zeta_{2}(N)$ and of a left ideal which is the direct sum of simple left ideals $L_{h}$ $(h \in H)$ of $N$ which are not of type $\left.2: N=\zeta_{2}(N) \oplus \underset{h \in H}{\oplus} L_{h}\right\}$.

Now, for every $h \in H, L_{h}$ is contained in the annihilator $\left(0: \zeta_{2}(N)\right)$ of $\zeta_{2}(N)$.

Indeed let $L$ be a simple left ideal and $x$ an element of $\zeta_{2}(N)$ such that the $N$-subgroup $L x$ is different from zero; then $L x$ is simple (being $N$-isomorphic to $L$ ). On the other side, $L x$ is contained in $\zeta_{2}(N)$, since $\zeta_{2}(N)$ is a left ideal. Thus $L x$ is $N$-isomorphic to a left ideal of type 2 (see [1], Proposition 2.2) and $L$ too must be of type 2 .

Looking next to the annihilator $\left(0: \zeta_{2}(N)\right)$, it can be remarked that it is the intersection of the annihizators of the left ideals $L$ of type 2 of $N$; in fact the inclusion $\left(0: \zeta_{2}(N)\right) \subseteq n(0: L)$ holds trivially, and the other is a consequence of $\zeta_{2}(N)$ being the direct sum of (a few of) the left ideals of type 2 and of the distributive property of direct sums .

Hence $\left(0: \zeta_{2}(N)\right)$ contains the intersection of the annihilators of all the $N$-groups of type 2 , that is the 2-radical $J_{2}(N)$ of $N$.

These assertions (which are true for every zerosymmetric near-ring) can be strengthened when $N$ is completely reducible, because every group of type 2 over such a near-ring is $N$-isomorphic to a left ideal of type 2 of $N$ (see [5], Corollary 3.11). Therefore

PROPOSITION 2. If $N$ is a completely reducible near-ring, the annihilator of $\zeta_{2}(N)$ is the 2-radical of $N$.

Then Proposition 1 can be rewritten as follows

PROPOSITION 3. A completely reducible near-ring $N$ is the direct sum of its socle of type 2 and of a left ideal which is contained in the 
2-radical $J_{2}(N)$ of $N$

\section{2. $\zeta_{2}$-decomposable near-rings}

The last result leads to study the intersection between $J_{2}(N)$ and $\zeta_{2}(N)$, in order to establish conditions under which $N$ is the direct sum of $\zeta_{2}(N)$ and $J_{2}(N)$. It seems useful to examine the question in a slightly more general context; call $\zeta_{2}$-decomposable a near-ring $N$ which has its socle of type 2 as a direct summand, and recall that $J_{2}(N)$ is contained in $\left(0: \zeta_{2}(N)\right)$.

Generally - as shown by the Counterexample 4 of section 3 - if $N$ is $\zeta_{2}$-decomposable but not completely reducible, $J_{2}(N)$ does not coincide with $\left(0: \zeta_{2}(N)\right)$; however they coincide restrictedly to $\zeta_{2}(N)$. In order to see this, let us prove

LEMMA 4. Let $N$ be a $\zeta_{2}$-decomposable near-ring. For each left ideal $C$ of $N$ such that $J_{2}(N) \subseteq C \subseteq\left(0: \zeta_{2}(N)\right)$, the intersection $\zeta_{2}(N) \cap C$ is the direct sum of all the left ideals of $N$ which are nilpotent and of type 2 .

Proof. Let $\zeta_{2}(N) \cap C=M$. All the nilpotent left ideals of $N$ are contained in $J_{2}(N)$ and therefore in $C$; among them, those of type 2 are necessarily contained in $\zeta_{2}(N)$; hence all the left ideals of $N$ which are nilpotent and of type 2 are contained in $M$. So it will be enough to show that there are nilpotent left ideals of type 2 of $N$ whose sum is the whole $M$.

Now, the $N$-group $M$ is the sum of its simple ideals $L_{i}$ since it is an ideal of the completely reducible $N$-group $\zeta_{2}(N)$ (see [5], Proposition 2.48). For each $L_{i}$ it results $L_{i}^{2}=(0)$ because

$$
L_{i} \subseteq M \subseteq C \subseteq\left(O: \zeta_{2}(N)\right) \subseteq\left(O: L_{i}\right)
$$


Furthermore, each $L_{i}$ is a left ideal of $N$ (since $M$ is a direct summand of the $N$-group $\zeta_{2}(N)$ and therefore of $N$ ) and is of type 2 , as it is a simple left ideal of $N$ contained in $\zeta_{2}(N)$ (see [1], Proposition 2.2).

Lemma 4 can be read as follows: in a $\zeta_{2}$-decomposable near-ming $N$ the left ideal $M=\zeta_{2}(N) \cap\left(O: \zeta_{2}(N)\right)$ coincides with $\zeta_{2}(N) \cap J_{2}(N)$. Besides, $M$ is nilpotent of class 2 and is zero if and only if $N$ has no nilpotent left ideal of type 2 .

From this statement the announced characterization follows.

PROPOSITION 5. A completely reducible near-ring $N$ is the direct sum of $\zeta_{2}(N)$ and $J_{2}(N)$ if and only if $N$ has no nilpotent left ideal of type 2 .

\section{Examples}

There are several classes of near-rings satisfying the condition expressed by Proposition 5.

EXAMPLE 1. Let $N$ be a completely reducible near-ring with right identity; then it is easily seen that $N$ is the direct sum of a finite number of simple left ideals, so that the intersection of all the maximal left ideals of $N$ is zero (see [5], Theorem 2.50). Such an intersection coincides with $J_{\frac{1}{2}}(N)$, for in a near-ring with right identity the maximal left ideals coincide with the O-modular ones. On the other side $J_{\frac{1}{2}}(N)$ contains every left nil ideal of $N$ (see [5], Theorem 5.37) and therefore $N$ has no nilpotent left ideal of type 2 . This proves

PROPOSITION 6. A completely reducible near-ming $N$ with might identity is the direct sum of $\zeta_{2}(N)$ and $J_{2}(N)$.

EXAMPLE 2. Let $N$ be a distributive near-ring completely reducible as a left $N$-group. Then $J_{2}(N)=J_{1}(N)=J_{0}(N)$ is a nilpotent ideal which is the direct sum of the annihilator $A(N)$ of $N$ and of the sum of the nilpotent left ideals of type 2 of $N$ (see [2], Theorem 6.1). Therefore 
PROPOSITION 7. A completely reducible distributive near-ring $N$ is the direct sum of $\zeta_{2}(N)$ and $J_{2}(N)$ if (and only if) $J_{2}(N)$ is the annihizator of $N$.

Observe that if the completely reducible near-ring $N$ is distributive, then $J_{2}(N)$ is nilpotent. This is also true of the 2-radical of a near-ring sum of its left ideals which are $N$-simple as $N$-groups (see [4], Theorem 2), but if $N$ is a general completely reducible near-ring, $J_{2}(N)$ may be non nilpotent. This can be seen in the nearrings of Example 1 or in the following

EXAMPLE 3. Let $N$ be the (external) direct sum of a field $F$ and of the near-ring $L$ built over the symmetric group $S_{3}$ denoted by (I) in [5], p. 410 .

Since the near-ring $L$ has no proper left ideal, the only left ideals of $N=F \oplus L$ are $F$ (which is the socle of type 2 of $N$ ) and $L$, which is the 2-radical of $N$ and non nilpotent because it contains idempotent elements.

Finally we show that there exist $\left(\zeta_{2}\right.$-decomposable $)$ near-rings $N$ such that $J_{2}(N)$ is properly contained in $\left(0: \zeta_{2}(N)\right)$.

EXAMPLE 4. Consider the dihedral group of order 12 , $D_{12}=\{a, b \mid 6 a=2 b=0, a+b=b+5 a\}$ and let $N$ be the distributive and commutative near-ring built over $D_{12}$ denoted by $N_{4}$ in [4]. The only ideal of type 2 of $N$ is $A=\{0,3 a\}$, so that $\zeta_{2}(N)=A$ and $\left(0: \zeta_{2}(N)\right)=\{0,2 a, 4 a, b, b+2 a, b+4 a\}$. Moreover $N=\zeta_{2}(N) \oplus\left(0: \zeta_{2}(N)\right)$; hence $N$ is $\zeta_{2}$-decomposable. However $J_{2}(N)=\{0,2 a, 4 a\}$.

\section{References}

[1] Stefania De Stefano and Simonetta Di Sieno, "On the type $v$ socles of a near-ring", Arch. Math. (Basel) 42 (1984), 40-44. 
[2] Stefania De Stefano and Simonetta Di Sieno, "Quasi-anelli distributivi completamente riducibili", Rend. Istit. Lomb. Acc. Sci. Lett. Cl. Sci. Ser. A (to appear).

[3] P. Lanusse Jones, "Distributive near-rings" (Thesis, University of Southwestern Louisiana, Louisiana, 1976).

[4] A. Oswald, "Completely reducible near-rings", Proc. Edinburgh Math. Soc. 20 (1976-77), 187-197.

[5] G. Pilz, Near-mings (North-Holland, Amsterdam, 1983).

Dipartimento di Matematica dell'Università di Milano, Via Saldini, 50 , 1-20133 Mi Iano Ml,

Italy. 